\title{
Canine IgA nephropathy: a case report
}

\author{
Akira YABUKI ${ }^{1) *}$, Takako SHIMOKAWA MIYAMA ${ }^{2)}$, Moeko KOHYAMA ${ }^{1)}$ and Osamu YAMATO ${ }^{1)}$ \\ 1) Laboratory of Veterinary Clinical Pathology, Joint Faculty of Veterinary Medicine, Kagoshima University, 1-21-24 Korimoto, \\ Kagoshima 890-8580, Japan \\ ${ }^{2)}$ Laboratory of Veterinary Internal Medicine, Joint Faculty of Veterinary Medicine, Yamaguchi University, 1677-1 Yoshida, Yamaguchi \\ 753-8515, Japan
}

(Received 20 March 2015/Accepted 7 November 2015/Published online in J-STAGE 21 November 2015)

\begin{abstract}
Immunoglobulin ( $\mathrm{Ig}$ ) A nephropathy is a rare form of canine glomerular disease. This report describes a case of canine IgA nephropathy showing characteristics typical of human IgA nephropathy. An 8-year-old, spayed female Miniature Dachshund showed persistent severe proteinuria without azotemia. She was receiving long-term glucocorticoid therapy due to chronic gastritis and an intra-abdominal suture granuloma. A renal biopsy demonstrated mesangial proliferative glomerulonephritis with predominantly mesangial IgA deposition and electron-dense deposits in the paramesangium. These findings closely resembled those of human IgA nephropathy. Glucocorticoid treatment was discontinued, and the angiotensin-converting enzyme inhibitor enalapril was administrated as an antiproteinuric agent. The proteinuria subsequently went into remission, and the patient has maintained good condition without recurrence.

KEY WORDS: angiotensin-converting enzyme inhibitor, canine, IgA nephropathy, proteinuria, renal biopsy
\end{abstract}

doi: 10.1292/jvms.15-0161; J. Vet. Med. Sci. 78(3): 513-515, 2016

Immunoglobulin (Ig) A nephropathy is a major cause of glomerular disease (GD) in humans [5], but is a rare form of GD in dogs [1]. There is one report documenting 3 clinical cases of canine IgA nephropathy [6], and another reporting marked glomerular deposition of IgA in 6 out of 100 canine autopsy cases [9]. However, the histopathological findings in those cases differed from the findings that characterize human $\operatorname{Ig}$ A nephropathy. We report a case of canine $\operatorname{IgA}$ nephropathy showing characteristics typical of human IgA nephropathy.

An 8-year-old, spayed female Miniature Dachshund, weighing $6.5 \mathrm{~kg}$, presented for evaluation of proteinuria. She had a history of chronic gastritis and intra-abdominal suture granuloma. She had received glucocorticoid (prednisolone) therapy for 7 months to treat the suture granuloma. Physical examination was normal, with no enlargement of tonsils. Urine was grossly normal, and microscopic evaluation of sediments revealed no increase of red blood cells, white blood cells, epithelial cells, casts or bacteria. Urine culture was negative. Severe proteinuria was found, and the urinary protein/creatinine ratio $(\mathrm{UP} / \mathrm{C})$ was 3.7 (reference range: $<0.5$ ); the UP/C was essentially unchanged 4 days later at 3.4 , but increased to 4.3 after one month. The complete blood count was unremarkable. The blood chemical analysis was also unremarkable, other than increased alkaline phosphatase (1831 U/l, reference range: $20-156 \mathrm{U} / l$ ) and gamma glutam-

*Correspondence to: Yabuki, A., Laboratory of Veterinary Clinical Pathology, Joint Faculty of Veterinary Medicine, Kagoshima University, 1-21-24 Korimoto, Kagoshima 890-8580, Japan.

e-mail:yabu@vet.kagoshima-u.ac.jp

C2016 The Japanese Society of Veterinary Science

This is an open-access article distributed under the terms of the Creative Commons Attribution Non-Commercial No Derivatives (by-nc-nd) License $<$ http://creativecommons.org/licenses/by-nc-nd/4.0/>. yltransferase (26 U/l, reference range: 1.2-6.4 U/l) activities. Both elevations had been noted before presentation, and cholangitis was also suspected as an underlying disease. The plasma urea nitrogen $(8.5 \mathrm{mg} / \mathrm{d} l$, reference range: $8-28 \mathrm{mg} /$ $\mathrm{d} l)$ and creatinine $(0.2 \mathrm{mg} / \mathrm{d} l$, reference range: $0.5-1.7 \mathrm{mg} /$ $\mathrm{d} l$ ) concentrations were normal. Hypoproteinemia was not present, and testing for antinuclear antibodies was negative. Systolic blood pressure measured by the Doppler method revealed mild hypertension (mean: $152 \mathrm{mmHg}$, reference range: $110-150 \mathrm{mmHg}$ ).

To give informed consent for a renal biopsy to definitively diagnose proteinuric kidney disease, the owner received information regarding risk, safeness, invasiveness and sample quality for each biopsy technique and agreed on a surgical method with a lateral small incision. Renal tissue samples were obtained from the left kidney using a $16-\mathrm{G}$ semi-automatic biopsy needle (Steri cut, TSK Laboratolry, Tochigi, Japan). The samples were processed for light microscopy (LM), transmission electron microscopy (TEM) and immunofluorescence (IF) analysis. The sample for LM was fixed in $10 \%$ neutral buffered formalin and embedded in paraffin routinely. The tissue was sectioned serially and stained with hematoxylin-eosin, periodic acid-Schiff, periodic acid methenamine-silver, Masson's trichrome and Congo red. The sample for TEM was fixed in $2.5 \%$ glutaraldehyde and embedded in epoxy resin (Quetol-812; Nisshin EM, Tokyo, Japan) routinely. Ultrathin tissue sections were stained with uranyl acetate and lead, and observed using a transmission electron microscope (H-7000KU; Hitachi, Tokyo, Japan). For IF microscopy, sections from snap-frozen tissues were cut with a cryostat. The sections were air-dried, washed in phosphate-buffered saline (PBS) and incubated in the primary antibodies. Goat polyclonal antibodies against dog $\operatorname{IgA}$, IgG, IgM and complement C3 (Bethyl Laboratories, Montgomery, TX, U.S.A.) were used as primary antibodies. After 

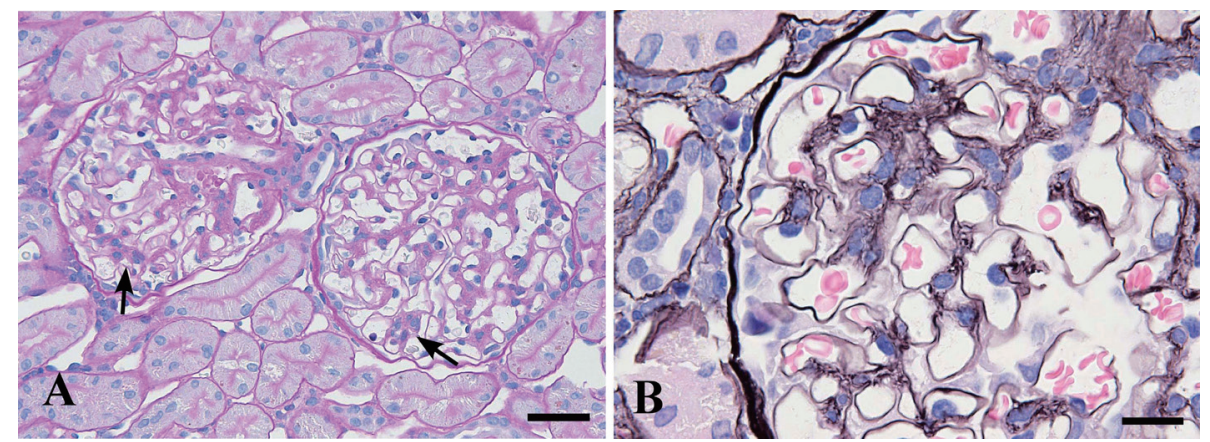

Fig. 1. Light microscopic findings of the renal biopsy sample. (A) Periodic acid-Schiff stain. A mild, diffuse increase of the mesangial area is observed in the glomeruli. The arrows indicate mesangial hypercellularity. (B) Periodic acid methenaminesilver stain. Membranous, membranoproliferative and sclerotic changes were not observed in the glomeruli. Bar: $20 \mu \mathrm{m}$.

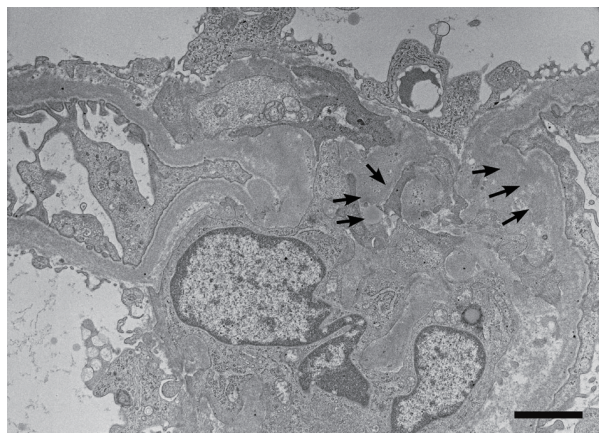

Fig. 2. Electron microscopic findings of the renal biopsy sample. The arrows indicate moderately electron-dense deposits in the paramesangium. Bar: $2 \mu \mathrm{m}$.

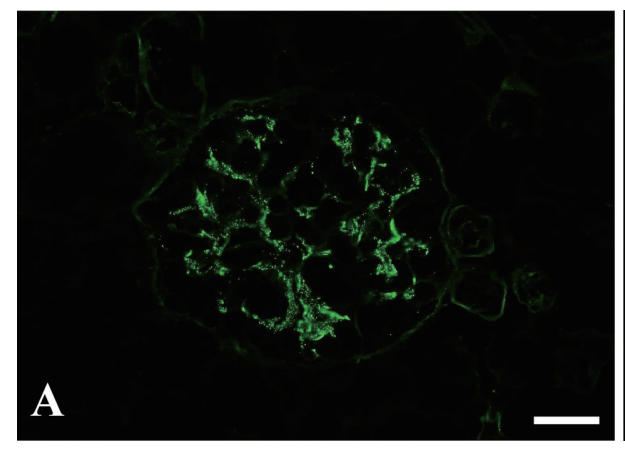

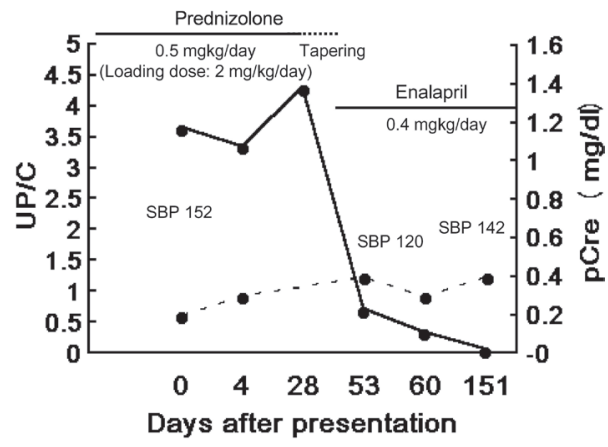

Fig. 4. Clinical course of the case. UP/C, urinary protein/creatinine ratio (solid line); pCre, plasma creatinine concentration (dotted line); SBP, systolic blood pressure $(\mathrm{mmHg})$. SBP was measured at 0,53 and 151 days after presentation.

Fig. 3. Immunofluorescent findings of the renal biopsy sample. (A) IgA. Granular, strong positive signals are observed in the mesangial area. (B) IgM. Positive signals are observed, but are weaker than those of IgA. Bar: $20 \mu \mathrm{m}$.

washing with PBS, the sections were incubated with Alexa Fluor 488-conjugated donkey anti-goat IgG (Life Technologies, Paisley, U.K.). To produce the negative control section, normal goat IgG (Santa Cruz Biotechnology, Santa Cruz, CA, U.S.A.) was used instead of a primary antibody. Stained sections were examined under a fluorescence microscope (BX-53, Olympus, Tokyo, Japan).

On LM, approximately 30 glomeruli were observed and showed a hallmark slight increase of the mesangial area, with or without mild mesangial hypercellularity (Fig. 1). These changes in the mesangial cells were seen in most of the glomeruli. No other remarkable glomerular or tubulointerstitial changes were observed. On EM, electron-dense deposits were observed in the paramesangium (Fig. 2). The foot processes of the podocytes were fused along a broad area of the glomerular basement membranes. On the IF images, 
granular, strong positive signals for IgA were detected in the mesangial area of all observed glomeruli (Fig. 3). Granular signals for IgM were also detected in the mesangium, but were much less intense than those for $\operatorname{IgA}$. No remarkable signals were observed for IgG and complement C3.

IgA nephropathy is difficult to diagnose in dogs, because IgA may be trapped nonspecifically in the glomeruli, regardless of the specific kidney disease [9]. Typical human IgA nephropathy shows mesangial proliferative glomerulonephritis with predominantly mesangial IgA deposition, and electron-dense deposits are frequently observed in the paramesangium [5]. Although several cases of canine IgA nephropathy have been reported previously, they showed membrano-proliferative glomerulonephritis, endocapillary proliferative glomerulonephritis and sclerosing glomerulonephritis instead of mesangial proliferative glomerulonephritis $[6,9]$.

The present case showed persistent severe proteinuria, which is a typical clinicopathological sign of canine GD, and the renal biopsy findings closely resembled those observed in human IgA nephropathy. We therefore diagnosed this patient with IgA nephropathy. If the renal biopsy findings are interpreted using the popular histologic grading system described by Haas et al. [4], the patient would be diagnosed with class I IgA nephropathy (minimal histologic lesion). In humans, the histological grade is well correlated with clinical outcome [3], and class I IgA nephropathy is known to have a good outcome [5]. The present case also showed a good outcome, as described below.

Glucocorticoids are commonly used to treat human GD, including IgA nephropathy. However, in dogs, glucocorticoid therapy for GD should be tailored to the conditions and underlying diseases of each case [8]. Although azotemia and worsened proteinuria are known risks of glucocorticoid therapy [2, 11], use of glucocorticoids might be appropriate if the case was fulminant or featured multisystem immunemediated disease. In the present case, glucocorticoid therapy was not indicated. The prednisolone treatment already being administered to treat intra-abdominal suture granuloma was therefore gradually discontinued while monitoring the condition of the dog, and no exacerbation of granuloma was observed after withdrawal. Angiotensin-converting enzyme inhibitors (ACEI) have well known beneficial effects in human proteinuric GD, including IgA nephropathy [10], and are a standard therapy in canine proteinuric GD [7]. In the present case, enalapril (Enacard, Merial Japan, Tokyo; $0.4 \mathrm{mg} / \mathrm{kg}$, once daily, oral) was administrated as an antiproteinuric agent. The proteinuria subsequently resolved, and the UP/C gradually decreased to $0.71,0.34$ and 0.06 at 53, 60 and 151 days after presentation, respectively (Fig. 4). The mild hypertension observed at first presentation was resolved; however, this could not be attributed to the antihypertensive effect of enalapril, as white-coat hypertension could not be ruled out in this case. At 17 months from initial presentation, the patient has maintained good clinical condition without any recurrence of proteinuria, development of azotemia or exacerbation of granuloma.

Although IgA nephropathy is considered the most com- mon form of primary GD in humans, it also develops secondary to numerous other conditions, such as hepatobiliary disease, gastrointestinal disease and infectious disease [5]. Hematuria, often associated with tonsillitis, is known to be a typical clinical feature. In dogs, a previous report suggested that gastrointestinal disease may be associated with IgA deposition in the glomeruli [9]. The present case had a history of chronic gastritis and cholangitis, and did not show hematuria or enlargement of tonsils. We therefore suspect that chronic gastritis and/or cholangitis may be associated with the development of secondary IgA nephropathy in the present case.

ACKNOWLEDGMENT. This research was supported by JSPS KAKENHI (Grant Number 25450446).

\section{REFERENCES}

1. Brown, C. 2011. Renal pathology. pp. 215-228. In: Nephrology and Urology of Small Animals (Bartges, J. and Polzin, D. J. eds.), Wiley-Blackwell, Ames.

2. Center, S. A., Smith, C. A., Wilkinson, E., Erb, H. N. and Lewis, R. M. 1987. Clinicopathologic, renal immunofluorescent, and light microscopic features of glomerulonephritis in the dog: 41 cases (1975-1985). J. Am. Vet. Med. Assoc. 190: 81-90. [Medline]

3. D'Amico, G. 2004. Natural history of idiopathic IgA nephropathy and factors predictive of disease outcome. Semin. Nephrol. 24: 179-196. [Medline] [CrossRef]

4. Haas, M. 1997. Histologic subclassification of IgA nephropathy: a clinicopathologic study of 244 cases. Am. J. Kidney Dis. 29: 829-842. [Medline] [CrossRef]

5. Haas, M. 2007. IgA nephropathy and Henoch-Schölein purpura nephritis. pp. 423-486. In: Heptinstall's Pathology of the Kidney, 6th ed. (Jennette, J. C., Olson, J. L., Schwaartz, M. M. and Silva, F. G. eds.), Lippincott Williams \& Wilkins, Philadelphia.

6. Harris, C. H., Krawiec, D. R., Gelberg, H. B. and Shapiro, S. Z. 1993. Canine IgA glomerulonephropathy. Vet. Immunol. Immunopathol. 36: 1-16. [Medline] [CrossRef]

7. IRIS Canine GN Study Group Standard Therapy Subgroup, Brown, S., Elliott, J., Francey, T., Polzin, D. and Vaden, S. 2013. Consensus recommendations for standard therapy of glomerular disease in dogs. J. Vet. Intern. Med. 27: S27-S43. [Medline] [CrossRef]

8. IRIS Canine GN Study Group Established Pathology Subgroup, Segev, G., Cowgill, L. D., Heiene, R., Labato, M. A. and Polzin, D. J. 2013. Consensus recommendations for immunosuppressive treatment of dogs with glomerular disease based on established pathology. J. Vet. Intern. Med. 27: S44-S54. [Medline] [CrossRef]

9. Miyauchi, Y., Nakayama, H., Uchida, K., Uetsuka, K., Hasegawa, A. and Goto, N. 1992. Glomerulopathy with IgA deposition in the dog. J. Vet. Med. Sci. 54: 969-975. [Medline] [CrossRef]

10. Reid, S., Cawthon, P. M., Craig, J. C., Samuels, J. A., Molony, D. A. and Strippoli, G. F. 2011. Non-immunosuppressive treatment for IgA nephropathy. Cochrane Database Syst. Rev. 16: CD003962. [Medline]

11. Waters, C. B., Adams, L. G., Scott-Moncrieff, J. C., DeNicola, D. B., Snyder, P. W., White, M. R. and Gasparini, M. 1997. Effects of glucocorticoid therapy on urine protein-to-creatinine ratios and renal morphology in dogs. J. Vet. Intern. Med. 11: 172-177. [Medline] [CrossRef] 